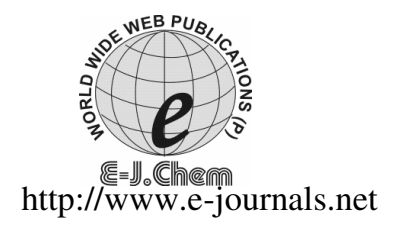

\title{
Synthesis of Nano Conducting Polymer Based Polyaniline and it's Composite: Mechanical Properties, Conductivity and Thermal Studies
}

\author{
M. BANIMAHD KEIVANI ${ }^{\S}, \mathrm{K}$ ZARE $^{\S}$, \\ M. AGHAIE, H. AGHAIE ${ }^{\AA^{*}}$ and M. MONAJJEMI ${ }^{\S}$ \\ ${ }^{\S}$ Department of Chemistry, \\ Islamic Azad University (IAU), Science \& Research Branch,Tehran, Iran. \\ Department of Chemistry, \\ Islamic Azad University (IAU), North Tehran Branch, Tehran, Iran. \\ keivani@acecr.ac.ir
}

Received 8 June 2009; Accepted 5 August 2009

\begin{abstract}
Polyaniline (PAn) was prepared chemically in the presence of bronsted acid from aqueous solutions. Polyaniline- nylon 6 composite (termed as PAn/Ny6) prepared via solvent casting method. The preparation conditions were optimized with regard to the mechanical properties of the polymer composite. It was found that the molar ratio of PAn to nylon have the greatest effect in determining the mechanical properties of polymer composite. Electrical conductivity was measured using standard method of four point probe. Spectrophotometric analysis (UV-Vis) was used for investigation of the effect of thermal treatment on polyaniline and it's composite.
\end{abstract}

Keywords: Polyaniline, Nano conducting polymer, Nylon 6, Conductivity, Thermal stability.

\section{Introduction}

Nano Conducting polymer films are a relatively new class of materials whose interesting metallic properties were first reported on 1977, with the discovery of electrically conducting polyacetylene ${ }^{1}$. This chance discovery occurred when a researcher accidentally added too much catalyst while synthesizing polyacetylene from acetylene gas, resulting in shiny metallic like substance rather than the expected black powder. This shiny semi-conducting material was subsequently partially oxidized with iodine or bromine vapors resulting in electrical conductivity values of up to $10^{5} \mathrm{~S} \mathrm{~cm}^{-1}$, which is in the metallic range $\mathrm{e}^{2}$. The importance of this discovery was recognized in 2000, when the Nobel prize for chemistry was awarded to the scientists who discovered ${ }^{3}$ electrically conducting polyacetylene in 1977. 
Among conducting polymers, a great deal of researches have been devoted to polyaniline (PAn) due to its good environmental stability, easy polymerization and low cost of monomer.

Polyaniline can be synthesized by the oxidation of aniline solution in bronsted acids by chemical oxidants or electrochemically ${ }^{4,5}$. In order to commercialize PAn, it is possible to fabricate blends or composites with some other conventional polymers. Polyamid (for example nylon) is a good choice because of its solubility in the same solvent of PAn (formic acid). Flexible and free standing films from composites of polyaniline- nylon (PAn/Ny) can be obtained by casting method ${ }^{6}$. Polyaniline (PAn) has been reported to have the following idealized structure (Figure 1$)^{7}$.

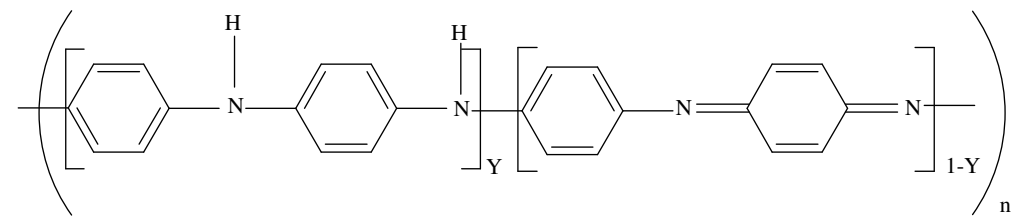

Figure 1. The general structure of PAn conducting polymers.

The structure containing $\mathrm{Y}$ reduced (benzenoid diamine) and (1-Y) oxidized repeat groups (quinoid diamine), where the oxidation state can be defined by the value of (1-Y). Y can be varied continuously in principle from one (the completely reduced material) to zero (to completely oxidized polymer). The completely reduced material $(\mathrm{Y}=1)$ is called leucoemeraldine and the completely oxidized form $(\mathrm{Y}=0)$ is termed as pernigraniline. Intermediate oxidation states, where $\mathrm{Y}=0.75$ and 0.25 are called protoemeraldine and nigraniline ${ }^{8}$. The true emeraldine oxidation state $Y=0.5$ of polyaniline in which $50 \%$ of the nitrogen atoms are protonated (consists of equal numbers of reduced and oxidized repeat units) is the most highly conducting (metallic) form of the polymer ${ }^{9}$. The relative content of the benzoid and quinoid forms depends on the acid concentration and the degree of oxidation of the polymer ${ }^{10}$. Chemical polymerization of aniline in aqueous acidic media can be easily performed using of oxidizing agents such as $\left(\mathrm{NH}_{4}\right)_{2} \mathrm{~S}_{2} \mathrm{O}_{8}$ is shown in the following Figure 2. Ammonium persulfate is the most popular and frequently used chemical oxidant for polymerization of aniline in acidic aqueous solutions.
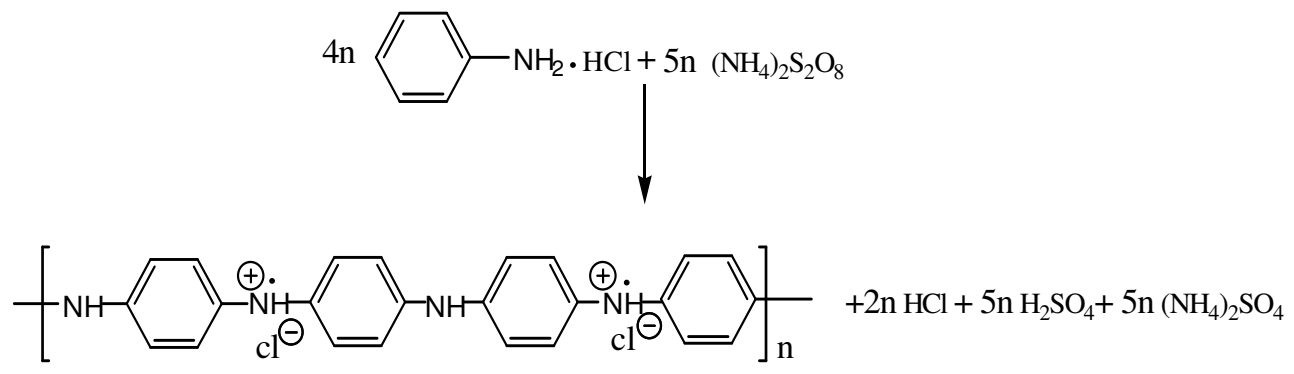

Figure 2. Overall polymerization reaction of PAn using ammonium persulfate.

Unfortunately, practical application of conducting polymers such as polypyrrole, polythiophene and polyaniline is limited particularly because of their poor mechanical properties. However, the composites prepared by mixing of conducting polymers with conventional polymers can be used in order to improve mechanical properties for all potential usages significantly ${ }^{11}$. 


\section{Experimental}

Aniline (Merck) was used after distillation. Nylon 6 (KN 136, Kopa co. Korea) was obtained as granules form. The other chemicals used were of high purity and AR grade.

Mechanical tests (Tensile strength) were carried out using S.D.L. Micro 250 (manufactured by Shirley Co. England). UV-Vis spectroscopy was carried out using UV2100 (Shimadzu). Electrical conductivity was measured using a home made 4-point probe device. A digital coulometer (ZAG chem. Iran) as current source, digital micrometer for measuring thickness of films and an accurate digital voltmeter (ALDA) for measuring potential during conductivity measurement were employed. Mechanical tests (Tensile strength) were carried out using S.D.L. Micro 250 (manufactured by Shirley co. England).

\section{Preparation of PAn and Pan/Ny6 composite}

$25 \mathrm{~mL}$ of Freshly distilled aniline $(0.2 \mathrm{M})$ was added into $500 \mathrm{~mL}$ of $2 \mathrm{M} \mathrm{HCl}$ in a beaker. $250 \mathrm{~mL}$ of $0.25 \mathrm{M}$ ammonium persulfate $\left(\mathrm{NH}_{4}\right)_{2} \mathrm{~S}_{2} \mathrm{O}_{8}$ solution, was added from a burette into the wellstirred monomer solution during $40 \mathrm{~min}$. Polymerization was carried out at $0 \sim 5{ }^{\circ} \mathrm{C}$. The molar ratio of monomer to oxidant was selected 1:1. The solution was continued stirring about $2 \mathrm{~h}$ after the complete addition of the oxidant. Then the green dark powder (polymer) was filtered, washed with dilute $\mathrm{HCl}$ solution, distilled water and methanol respectively in order to remove impurities and remainder monomers. Then the product was dried at $50-60{ }^{\circ} \mathrm{C}$ in an oven for $2 \mathrm{~h}$ at ambient conditions. The synthesized polymer powder $(\mathrm{PAn} / \mathrm{HCl})$ was treated with a solution of ammonia $(0.5 \mathrm{M})$ for 4 hours at room temperature in order to change the polymer into emeraldine base (EB) state, which is the only processable form of polyaniline. PAn composite films were then prepared using co-dissolution method employing formic acid (88\%) as solvent. For fabrication of PAn/Ny composites, EB and nylon 6 were separately dissolved in formic acid (1\% w/v). Then they were mixed together with different proportions. Free standing films $(40-50 \mu \mathrm{m})$ were prepared by casting these solutions in petri dishes, placed in an oven at $60{ }^{\circ} \mathrm{C}$ overnight. The films obtained were removed from petri dishes by immersion in dilute $\mathrm{HCl}$ solution for few minutes. The membranes dried at room temperature after washing with distilled water.

\section{Results and Discussion \\ Conductivity and morphology}

Electrical DC-conductivity of PAn composite films (doped with $\mathrm{HCl}$ ) was measured using conventional 4-point probe technique ${ }^{12}$. In order to measure conductivity, the polymer samples were cut into $1 \times 4 \mathrm{~cm}$ strips for conductivity. Conductivity measurements were carried out after doping the polymer samples with $0.2 \mathrm{M} \mathrm{HCl}$ solution for $2 \mathrm{~h}$ at room temperature after drying at ambient conditions. Conductivity of $\mathrm{PAn} / \mathrm{Nylon}$ composites after doping with $\mathrm{HCl}$, was much lower than pure PAn but is much higher than nylons (insulator). The highest conductivity for PAn/Ny6 was $0.009 \mathrm{~S} / \mathrm{cm}$ in weight ratios of 1:2. With increasing the percentage of nylon, conductivity of polymer composite $\sigma(\mathrm{S} / \mathrm{Cm})$ decreased gradually (Table 1$)$.

$\mathrm{PAn} / \mathrm{Ny} 6$ composites were changed into insulator when treated with a dilute ammonia solution $(0.2 \mathrm{M})$. The conductivity was recovered completely when redoped with $\mathrm{HCl}$ solution $(0.2 \mathrm{M})$. It was also found that chemical treatment of PAn/Ny6 composites films with chemical reductants such as sodium thiosulfate and oxidants such as potassium permanganate leads to increasing resistivity of polymers significantly. The conductivity was recovered when the samples were redoped in a dilute $\mathrm{HCl}$ solution. For doping of $\mathrm{PAn} / \mathrm{Ny}$ composite dilute solution of $\mathrm{HCl}$ was employed since nylons are not usually chemically stable in strong $\mathrm{HCl}$ solution because of hydrolysis reaction. As our results showed conductivity of the polymer is changed when treated in different media and the changes are reversible. 
Table 1. Electrical conductivity of PAn/Nylon6 composite prepared in different weight ratio.

\begin{tabular}{ccc}
\hline No. & weight ratio PAn : Ny 6 & $\sigma, \mathrm{S} / \mathrm{Cm} \times 10^{-3}$ \\
\hline 1 & $1: 2$ & 9 \\
2 & $1: 3$ & 7 \\
3 & $1: 4$ & 5 \\
\hline
\end{tabular}

Characterisation of PAn and PAn/Ny6 composite was also carried out using scanning electron microscopy (SEM). All the electron micrographs were obtained from powder (for PAn and EB form) and film (for PAn/Ny6) specimens of this materials. SEM observations have shown the differences in morphology of PAn (or EB form) and PAn/Ny6 composites. The SEM pictures are given in Figure 3.

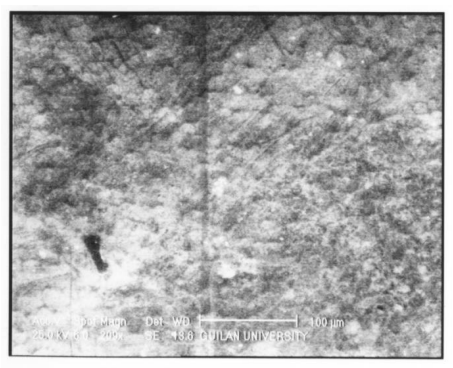

$\mathbf{a}$

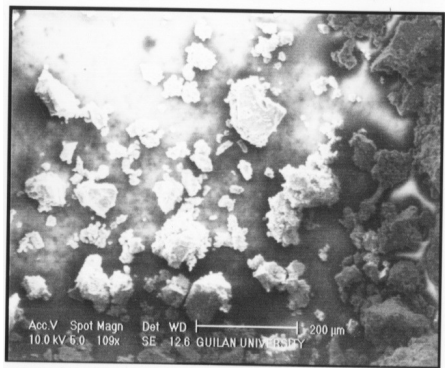

c
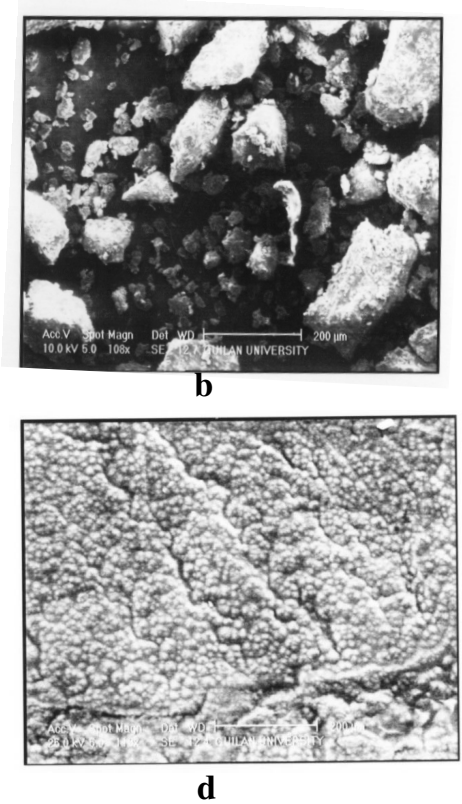

Figure 3. Scanning electron micrographs (SEM) of (a) EB/Ny6, (b) EB powder,

(c) $\mathrm{PAn} / \mathrm{HCl}$ powder, (d) $\mathrm{EB} / \mathrm{Ny} 6, \mathrm{HCl} 0.2 \mathrm{M}$ was used for doping of the polymer.

\section{Mechanical properties}

In order to perform mechanical test, polymer samples were prepared as free standing films using casting technique. Free standing films with adequate mechanical properties of PAn composites with nylons can be easily prepared by casting from formic acid. The results obtained in this investigation have been summarized in Table 2.

Table 2. Mechanical properties of PAn /Nylon 6 composite.

\begin{tabular}{ccccc}
\hline No. & Weight ratio PAn : Nylon 6 & $\delta \mathrm{L}, \mathrm{mm}$ & $\mathrm{F}(\mathrm{N})$ & Tensile strength (MPa) \\
\hline 1 & $1: 2$ & 0.20 & 2.4 & 6 \\
2 & $1: 3$ & 0.30 & 8.4 & 19 \\
3 & $1: 4$ & 0.7 & 1.4 & 3 \\
4 & Pure Ny6 & 1.53 & 6.4 & 15 \\
\hline
\end{tabular}




\section{Thermal stability studies}

In order to find out the effect of thermal treatment on electrical conductivity and also to investigate the reversibility of conductivity upon thermal treatment, the polymer samples (PAn/Ny 6) were prepared as a thin film deposited on the surface of lambs. It was observed that when the polymer was thermally treated, its dry state conductivity was increased upon thermal treatment up to $100{ }^{\circ} \mathrm{C}$ and upon subsequent cooling, conductivity back to its initial value.

Effect of thermal treatment on the polymer structure was also studied using UV-Vis analysis (Figure 4). As our results show PAn (EB form) is thermally stable up to $130^{\circ} \mathrm{C}$ under air atmosphere. Some minor structural changes (without decomposition) may occur even after mild heat treatment but not any important changes were observed in the UV-Vis spectrum of the polymer before and after heat treatment up to $130{ }^{\circ} \mathrm{C}$. However, when the polymer is treated at more elevated temperatures $\left(\mathrm{T}>150^{\circ} \mathrm{C}\right)$, some irreversible changes such as crosslinking might be occurred. In the case of PAn/Nylon6 polymer composites showed more thermal stability (up to $180{ }^{\circ} \mathrm{C}$ ) in air than pure PAn (Figure 5).

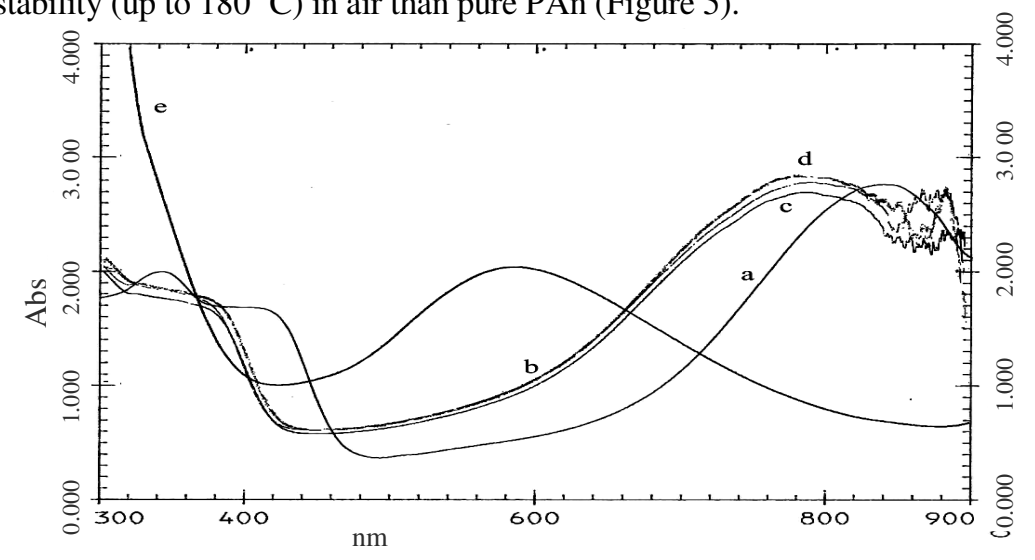

Figure 4. UV-Vis spectra of PAn (EB); (a) Before thermal treatment, (b) After heat treatment at $80{ }^{\circ} \mathrm{C}$, (c) After heat treatment at $100{ }^{\circ} \mathrm{C}$, (d) After heat treatment at $130{ }^{\circ} \mathrm{C}$, (e) After heat treatment at $150{ }^{\circ} \mathrm{C}$, the time of exposure at maximum temperature was $30 \mathrm{~min}$.

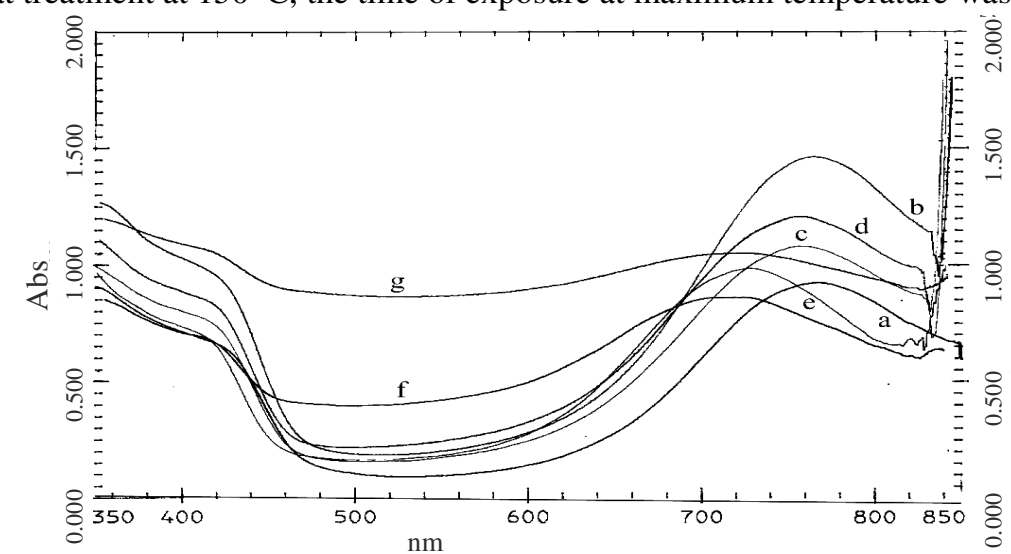

Figure 5. UV-Vis spectra of PAn (EB)/Nylon 6, (a) Before thermal treatment, (b) After heat treatment at $80{ }^{\circ} \mathrm{C}$, (c) After treatment at $100{ }^{\circ} \mathrm{C}$, (d) After heat treatment at $130{ }^{\circ} \mathrm{C}$, (e)After heat treatment at $150{ }^{\circ} \mathrm{C}$, (f) After heat treatment at $200{ }^{\circ} \mathrm{C}$, (g) After heat treatment at $250{ }^{\circ} \mathrm{C}$. The conditions were the same as Figure 4. 


\section{Conclusion}

Polyaniline and its composites with conventional polymers such as nylons can be easily processed from formic acid via cast method $\left(\mathrm{bp}=105^{\circ} \mathrm{C}\right)$ producing desired thickness of the film. It was found the percentage of the nylon 6 has very important effect on mechanical, electrical and thermal stability of the polyaniline composite. The best weight ratio for PAn/Nylon6 was found to be 1:3 with regard to mechanical. Electrical conductivity is decreased with increasing of the nylon percentage in polymer composite. According our $\mathrm{UV}$-vis investigation the polymer composites are more thermally than pure polyaniline.

\section{References}

1. Shirakawa H, Louis E J,Mac Diarmid A G, Chiang C K and Heeger A J, J Chem Soc; Chem Commun., 1977, 578.

2. Herbert Naarmann, Polymers to the Year 2000 and Beyond, John Wiley \& Sons, 1993, Chapter 4.

3. Wallace G G and Spinks G M, Conductive Electroactive Polymers; Intelligent Material Systems, $2^{\text {nd }}$ Edn., CRC Press, 2003, 1.

4. Angelopouls M, Austurias G E and Ermer S P and Ray A, Mol Cryst., 1988, 160, 151-163.

5. Pasquali M, Pistoia G and Rosati R, Synthetic Metals, 1990, 53, 1-15.

6. Malmong J A and Compoli C S, Synthetic Metals, 2001, 119, 87-88.

7. Ansari R, E J Chem., 2006, 3(4), 186-201.

8. Angelopoulos M, Rarnd A, MacDiarmid A G and Epstein A J, Synthetic Metals, 1987, 21, 21-30,

9. MacDiarmid A G, Richter J C, Somasiri A F and Epstien A G, in Conducting polymers, (Ed.L.Alcacer), Reidel Publishing Co. Dordrecht, 1987, 105.

10. Bhadani S N, Gupta M K and Gupta S K S, J Appl Polym Sci., 1993, 49, 397-403.

11. Ansari R, Price W E and Wallace G G, Polymer, 1996, 37(6), 917-923.

12. ASTM, Designation: D 4496. 


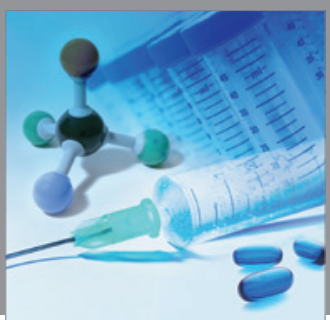

International Journal of

Medicinal Chemistry

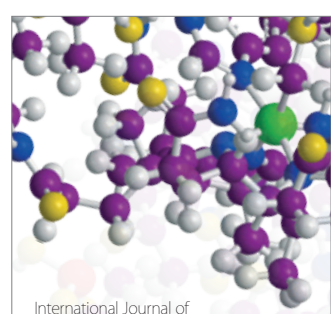

Carbohydrate Chemistry

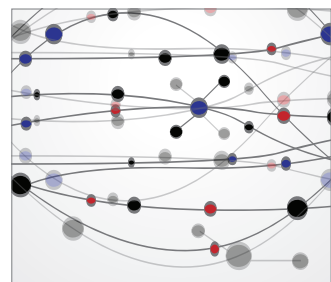

The Scientific World Journal
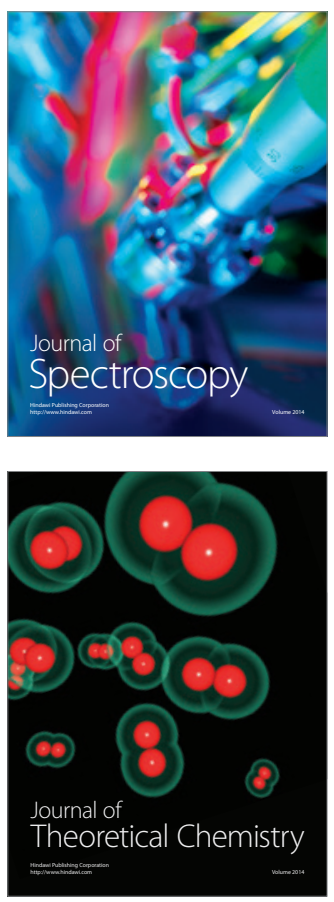
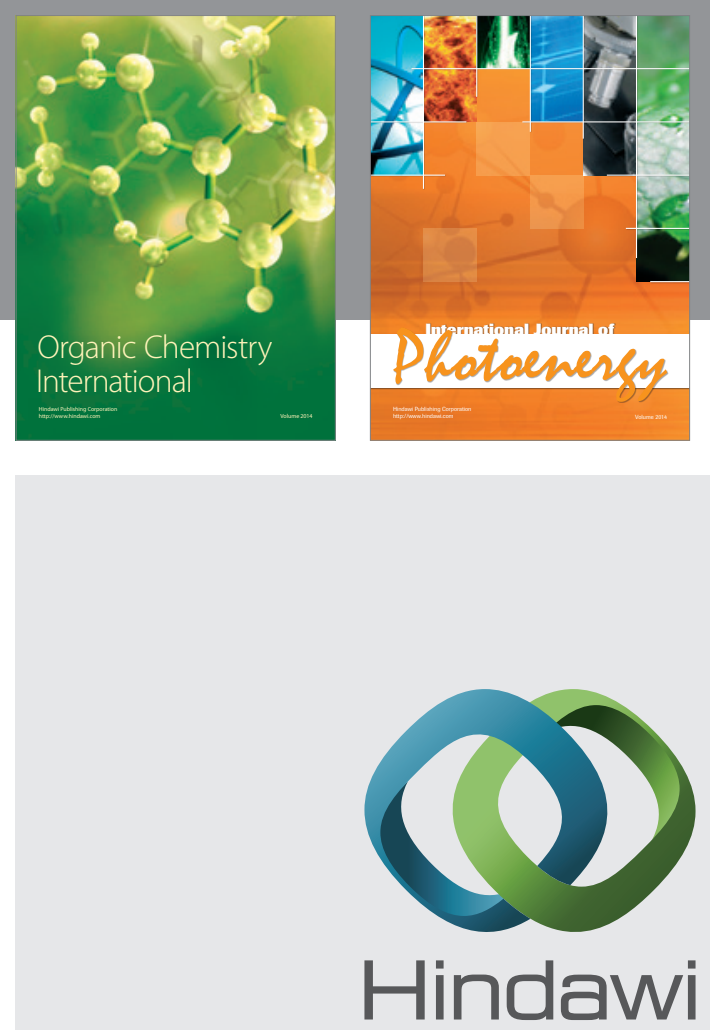

Submit your manuscripts at

http://www.hindawi.com
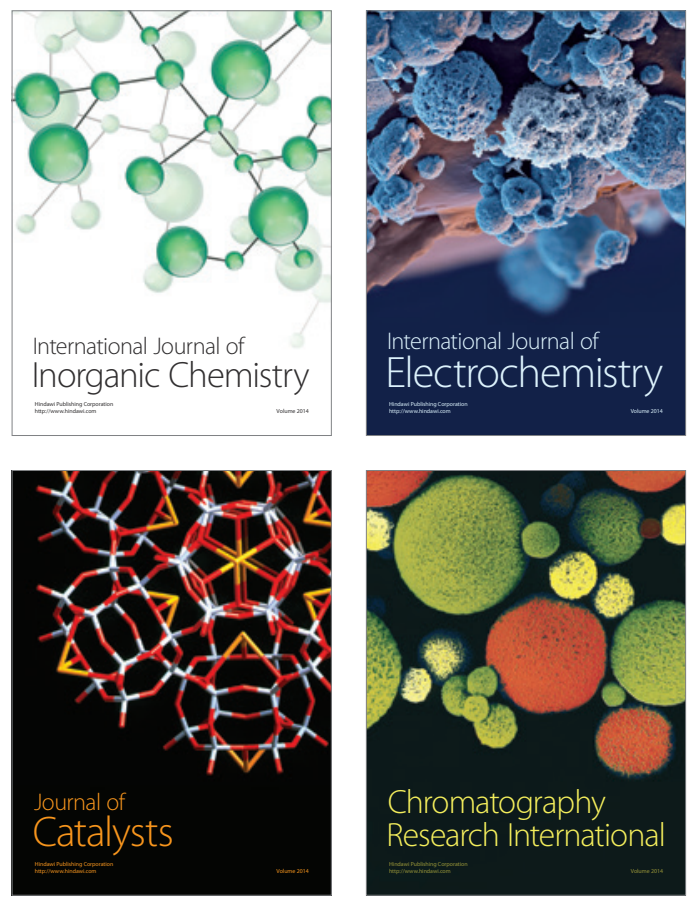
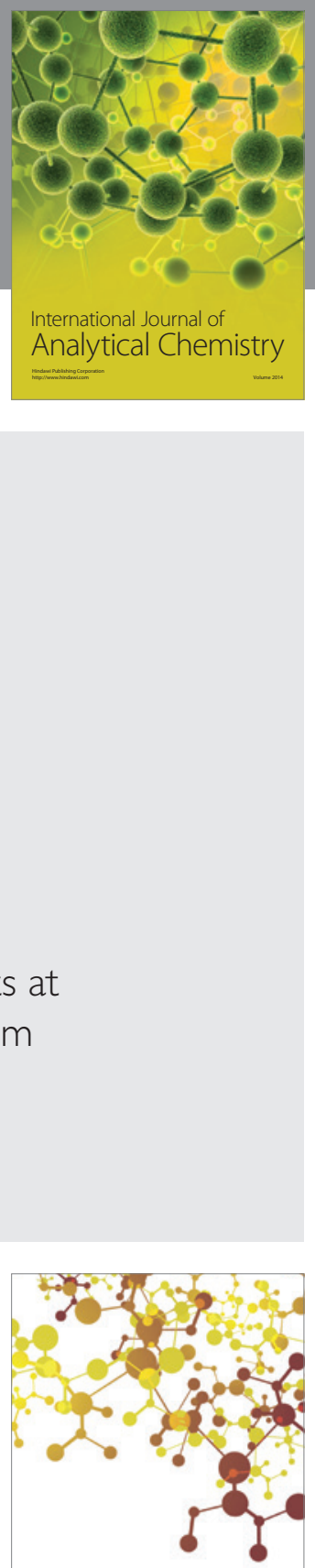

Journal of

Applied Chemistry
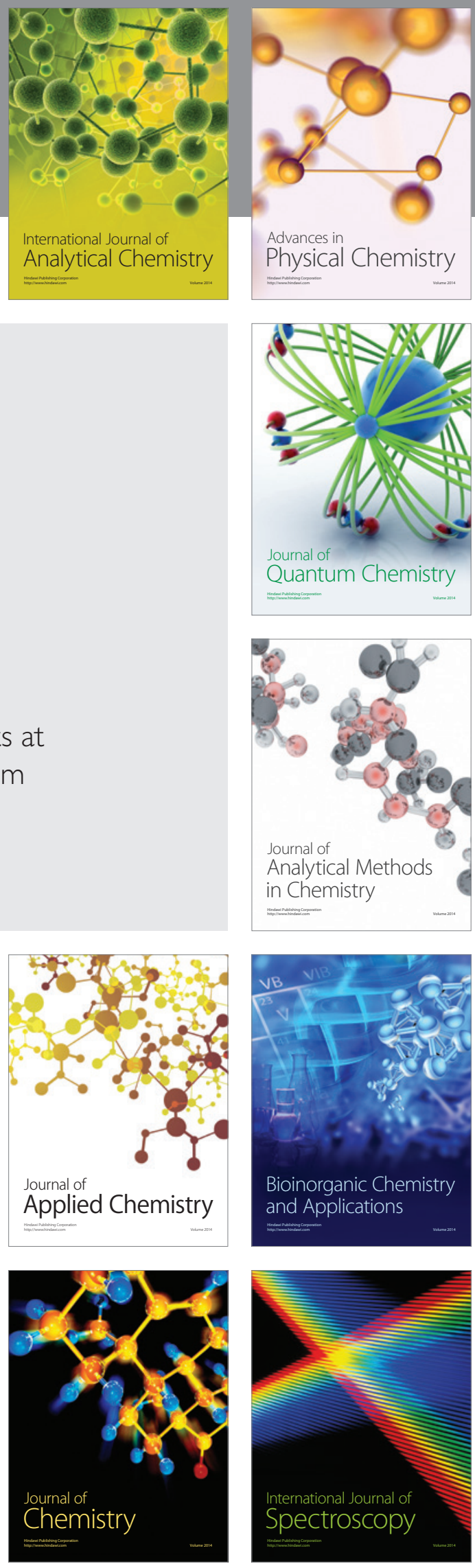